\title{
Evolution of phenotypic variance: non- Mendelian parental influences on phenotypic and genotypic components of life-history traits in a generalist herbivore
}

\author{
YVES CARRIÈRE \\ Centre for Pest Management and Behavioural Ecology Research Group, Department of Biological Sciences, Simon \\ Fraser University, Burnaby, BC, Canada V5A $1 S 6$
}

\begin{abstract}
Non-Mendelian parental effects are a form of phenotypic plasticity that influences offspring development and modifies their phenotype. They could have major evolutionary impact by changing the process of selection and the correspondence between offspring genotypes and phenotypes. To examine the influence of parental effects on life-history traits in the obliquebanded leafroller, Choristoneura rosaceana (Harris), three successive generations were raised in a constant laboratory environment, the first and subsequent generations being produced by field-raised and laboratory-raised parents, respectively. Between-generation variation in phenotypic means, variances and among- and within-family variance components were used to assess the magnitude of the influence of parental effects on allelic expression. The differences between parent-offspring regressions derived using parents from the first and second generations were used to estimate the directionality of the influence of parental effects on the family means of the traits.

It appears that parental effects influenced the mean and variance of life-history traits at population and family levels. For development time and within-family diapausing propensity, the families of parents developing in a specific environment (laboratory or field) were apparently affected by a similar parental effect. For larval growth rate and pupal weight, it appears that different parental effects transmitted by field parents influenced the family means. Consequently, parental effects transmitted by field parents did not change the correspondence between the offspring phenotypic and breeding values in the first two traits but modified this relationship in the later traits. Parental effects influenced simultaneously the range of phenotypes produced by a genotype and its mean phenotype. Therefore, they may represent a major developmental mechanism by which organisms adapt to unpredictable and predictable environments.
\end{abstract}

Keywords: bet-hedging, heritabilit,, life-history traits, maternal effects, phenotypic plasticity, Tortricidae.

\section{Introduction}

It has long been recognized that environmentallyinduced phenotypic variation, or phenotypic plasticity, has important implications for life-history evolution (Schmalhausen, 1949; Bradshaw, 1965; Levins, 1968). On the basis of the kind of selection promoting phenotypic variation, it is currently possible to distinguish between two types of models that describe the evolution of phenotypic plasticity expressed in discrete environments. The first category of models describes

Correspondence: Department of Biology, McGill University, 1205 Dr Penfield Avenue, Montreal, Quebec, Canada H3A 1 B1. the evolution of repeatable changes in the mean phenotype expressed by a genotype across environments, i.e. of the average norm of reaction in a population /Via \& Lande, 1985; Via, 1987, 1993; van Tienderen, 1991; Gomulkiewicz \& Kirkpatrick, 1992). These models assume that reaction norms evolve as a result of stabilizing selection operating in each environment. The second category of models describes the evolution of within-genotype (family, inbred line or clone) variation expressed in discrete environments (Kaplan \& Cooper, 1984; Bull, 1987; Seger \& Brockman, 1987). In these models, a developmental mechanism is postulated to act within each genotype as a phenotypic 'randomization' process. The simplest models assume that the 
environmental components determining the phenotype of individuals are uncorrelated with environmental cues (the fair 'coin-flipping' strategy of Kaplan \& Cooper, 1984). The developmental mechanism, however, is assumed to be sensitive to environmental variation (or developmental noise), such that the magnitude of the range of phenotypes produced by a genotype may differ in discrete environments. In addition to assuming stabilizing selection, these models also consider cases where the optimal phenotype fluctuates unpredictably in specific environments.

In either type of model, genotypic variation in expression of the phenotype across environments is needed for an optimal phenotype to evolve in each environment. Genetic variation in norms of reaction is believed to occur because alleles contribute in a different way to the phenotype in each environment or because environmental effects cause different loci to be expressed in each environment (Via \& Lande, 1985; Via, 1993; C. D. Schlichting \& M. Pigliucci, unpublished data). Variation in within-genotype phenotypic variance is believed to arise from differences in the sensitivity of genotypes to specific environmental effects (Kaplan \& Cooper, 1984; Bull, 1987). Although the developmental mechanisms generating phenotypic plasticity are still poorly understood, it is becoming increasingly clear that phenotypic plasticity can modulate the expression of genetic variation in, and covariation among, life-history traits (Gupta \& Lewontin, 1982; van Noordwijk \& Gebhardt, 1987; Newman, 1988; Scheiner et al., 1989; Stearns, 1989, 1992; Holloway et al., 1990). Therefore, a greater understanding of the impact of development on these genetic parameters may help to circumscribe the utility of current evolutionary models that use genetic variances and covariances to predict the dynamics of phenotypic changes in populations (e.g. Lande, 1979; Via \& Lande, 1985). A better appreciation of the role of development in modulating genetic variation and covariation may also lead to the elaboration of more realistic models describing the evolution of phenotypic plasticity (Stearns, 1992; C. D. Schlichting \& M. Pigliucci, unpublished data).

Non-Mendelian parental effects (often designated as maternal effects) are another source of factors that can influence or regulate the expression of alleles and induce phenotypic changes in a genotype (the offspring). Within the framework of quantitative genetics, these effects are considered to be environmental in nature (Falconer, 1981; Kirkpatrick \& Lande, 1989). Parental effects often produce phenotypic changes that appear to be adaptive in response to spatially and temporally heterogeneous environments (Mousseau \& Dingle, 1991a, b). In this sense, they may be compared to phenotypic plasticity induced by purely environ- mental factors, because they can modify the process of phenotypic selection operating on the offspring (genotype). Parental effects can also influence the evolutionary response to selection if they change the relationship between the phenotypes of the individuals of a family and their breeding values, if they change the genetic covariance between traits expressed in different environments, or if they introduce time lags in the response to selection (Falconer, 1981; Groeters \& Dingle, 1987; Kirkpatrick \& Lande, 1989).

Parental effects can take many forms (Kirkpatrick \& Lande, 1989; Mousseau \& Dingle, 1991a). This paper considers one type of such transgenerational effects: the non-Mendelian factors supplied by the parents to the egg that can modify offspring development. Although parental effects are more frequently transmitted by mothers than fathers (Kirkpatrick \& Lande, 1989; Mousseau \& Dingle, 1991a), it is important to note that paternal effects can also influence the phenotype of their offspring (Giesel, 1986, 1988).

The phenotypic modifications caused by parental effects can be qualitative, as in maternally induced polyphenism producing sexual or asexual forms of aphids (Hardie \& Lee, 1985). Parental effects can also cause quantitative changes in the mean or variance of the traits. Quantitative parental effects can be categorized according to the source of variation generating the effects. The first type occurs when the mean phenotype of the progeny (or the within-progeny variance) is a function of the phenotype of the parents (Janssen et al., 1988; Kirkpatrick \& Lande, 1989). The second type refers to modifications influencing the offspring independently of the parental phenotypes. These latter effects can be determined by the environmental conditions experienced in the parental generation (e.g. the parental photoperiodic, climatic or nutritional conditions), by the physiological state of the parents (e.g. parental age) or by genetic variation among the parents in the production of parental effects (Mousseau, 1991; Mousseau \& Dingle, 1991a, b). Parental effects of this second type have been separated on the basis of their capacity to influence the among-family or within-family variance (the type 1 and type 2 parental effects of Rossiter (1991)).

Although the influence of parental effects on the phenotypic mean of populations and on variance in the offspring of individuals is well documented, detailed information on their effect on the expression of alleles is rare. This is surprising if one considers the abundance of parental effects. Of specific interest is the consequence on genetic variance components of parental effects that induce variation in the range of phenotypes produced by a genotype. In this paper, I present the results of a quantitative-genetic analysis that investigated whether parental effects have a significant 
influence on the life-history traits of the obliquebanded leafroller, Choristoneura rosaceana (Harris). The specific objectives were to describe the type of parental effects involved, their impact on the populational mean and variance, on the expression of amongand within-genotype variation and on the heritability estimates of life-history traits.

\section{Materials and methods}

\section{C. rosaceana life-cycle}

C. rosaceana is a polyphagous tortricid which can use a variety of deciduous trees, shrubs and even conifers as hosts (Chapman \& Lienk, 1971; Carrière, 1992). In the Okanagan valley of British Columbia where this experiment was conducted, C. rosaceana exhibits two generations per summer (i.e. is bivoltine). The overwintering larvae resume feeding in the spring and the first adult flight is initiated in June. A proportion of the larvae produced by each female of the first flight initiate diapause in their third instar (Carrière, 1992; Carrière \& Roitberg, in press) whereas the rest of the caterpillars develop to adulthood and produce a second flight in August. All fall-produced larvae initiate diapause in their second or third instar (Carrière, 1992). A life-history involving a mixture of active and dormant phenotypes within a generation is common in arthropods and plants that exploit uncertain environments (Rausher, 1985; Seger \& Brockman, 1987; Mousseau \& Dingle, 1991b). As in the case of $C$. rosaceana (Carrière, 1992; Carrière \& Roitberg, in press), such phenotypic variation has often been interpreted as an adaptive bet-hedging strategy.

\section{Experimental approach}

Three consecutive generations of $C$. rosaceana were raised in the laboratory in a constant environment. Offspring of the first generation were produced by field-raised parents. The two subsequent generations were derived from parents sampled from the previous, laboratory-raised generation. The large number of families $(n=42)$ used to establish each generation and the low mortality under the rearing conditions ensured minimal change in gene frequency from generation one to three (see Discussion). Consequently, betweengeneration variation in the mean and variance of the traits and in the among- and within-family variance can be attributed to differences in parental effects.

\section{Experimental rationale}

For individuals developing in a homogeneous environment, the phenotypic value of a trait is determined by the sum of an additive genetic component, a component including the environmental and non-additive effects, and a parental effect (assuming additivity of these sources of variation; Falconer, 1981). Furthermore, variation in parental effects may be due to genetic or environmental differences in the parental generation (Mousseau \& Dingle, 1991a). Assuming that the parental effects are independent of the parental phenotype and transmitted by a single sex, the variance in parental effects can be expressed as: $V_{\mathrm{pp}}=V_{\mathrm{gp}}+V_{\mathrm{ep}}+V_{\mathrm{ip}}$, where $V_{\mathrm{gp}}$ is the genotypic variance in parental effects (owing to variation in the average response of parental genotypes exposed to the same environmental conditions), $V_{\text {ep }}$ is the environmental variance generated when the parents are influenced by different conditions and $V_{\text {ip }}$ is the variance resulting from residual deviations of a microenvironmental and individual nature (this term includes within-progeny variation generated by differences in allocation of the parental effects; see Rossiter (1991)).

The contribution of the $V_{\text {ep }}$ term depends on how the environment is subdivided with respect to the factor(s) generating the parental effects. If every parent within a population is exposed to identical conditions, the $V_{\text {ep }}$ term will be 0 and each family mean will be influenced in the same way by the environmental component of the parental effects. Therefore, even if a parental effect were transmitted, this type of environmental structure is not expected to change the correspondence between the phenotype and breeding value of the offspring. If the parents develop in heterogeneous environments, however, the $V_{\text {cp }}$ term may differ from zero. Accordingly, the phenotype and breeding value of the offspring may be influenced independently. This leads to the prediction that the phenotypes of parents and offspring raised in a constant environment are expected to be less correlated when the grandparents have been raised in different rather than in similar environments. Note that this interpretation is based on the assumption that the relative contribution of the $V_{\mathrm{gp}}$ term is identical in field- and laboratory-reared genotypes (i.e. there is no 'crossing' $V_{\mathrm{gp}} \times$ environment interaction).

\section{Predictions}

Three types of evidence will be used to support the hypothesis that parental effects are present in $C$. rosaceana. (1) A significant change in the mean and variance of the traits between the first and subsequent generations. (2) A significant change between the first and subsequent generations in the among- and withinfamily variance. Such a change would suggest a differ- 
ent contribution of the field and laboratory parents to the $V_{\text {ep }}$ and $V_{\text {ip }}$ terms, respectively. (3) Lower parent-offspring heritability estimates obtained when the offspring had field-raised grandparents than when they had laboratory-raised grandparents.

\section{Source of insects and experimental procedures}

Larvae in their last two instars were collected from the population described in Carrière (1992) on two consecutive days at the end of May 1990. They were transferred to an artificial pinto bean diet (modified from Shorey \& Hale, 1965) and raised in the laboratory under a $16 \mathrm{~L}: 8 \mathrm{D}$ light regimen and a $22.5 \pm 1^{\circ} \mathrm{C}$ (day), $20 \pm 1^{\circ} \mathrm{C}$ (night) temperature cycle. These conditions were used to raise all insects in this experiment.

Resulting adults were paired to yield 42 families of larvae that made up the first generation. Forty-two families were also raised in the second and third generations. To increase the precision of the estimates of heritability for diapausing propensity, the parents of the second and third generations were mated assortatively with respect to that trait. Sib-matings were not permitted and a given female was only mated to one male. A family consisted of 30 larvae, each caterpillar being raised in an individual cup. The larvae always originated from the mother's first egg mass. Each family was placed as a group in a randomly chosen section of the growth chamber and every day the groups were randomly re-located to minimize microclimatic influences on $C$. rosaceana growth.

\section{Measurement of the life-history traits}

Traits measured were pupal weight (PW), total development time (DT), larval live weight after 16 days of feeding (LW) and the proportion of larvae initiating diapause within a family (DIAP). In the field, pupal weight is positively correlated with total fecundity in $C$. rosaceana (Carrière, 1992). A discussion of the relationship between fitness and variation in DIAP can be found elsewhere (Carrière \& Roitberg, in press).

A larva was considered to be in diapause if after 16 days it had spun a cocoon within its cup or it was in the beginning of its third instar or smaller (at that time, most larvae were in their fifth or sixth instars). Sixtyeight per cent of these smaller larvae successfully spun a cocoon and diapaused when given access to a diapausing site (as in Carrière, 1992). This is comparable to the diapausing incidence of larvae that were fed on different host plants (Carrière, 1992) and suggests that the criteria used to designate diapausing larvae were adequate.

\section{Influence of parental effects on the mean and variance of the traits}

The distributions of DIAP within the parental families of a given generation and the families of the next generation were compared. The distribution of DIAP within the first experimental generation produced by spring parents was also compared with the distribution of DIAP within families produced by summer adults, originating from larvae collected in August 1990. These latter larvae were collected in their last two instars from the same orchard sampled to establish the first experimental generation.

\section{Experimental analysis and estimations of heritabilities}

Diapausing intensities. It was assumed that DIAP is an 'all-or-none' trait that conforms to a model describing quantitatively inherited threshold characters (Falconer, 1981). Such a model has been found to describe adequately the inheritance of many characters of biological interest (Roff, 1986, in press; Tauber \& Tauber, 1986).

The heritability of DIAP was estimated using a parent-offspring regression method (Roff, 1986) where the regressions are derived using probit-transformed family values. No larvae initiated diapause in four and two families of the second and third generations. These families were excluded from the analyses as a proportion of 0 is not probit transformable. The heritability estimates obtained using regressions of offspring on single parental values were corrected for assortative mating (Falconer, 1981; Roff, 1990).

Larval weight, pupal weight and development time. For each generation, the among- and withinfamily variance in each trait was estimated (VARCOMP procedure; SAS, 1988). Between-generation variation in the magnitude of the variance components was investigated for each trait using two-tailed $F$-tests (Sokal \& Rohlf, 1981). Full-sib and parent-offspring heritability estimates of the traits were obtained for each sex and their standard errors calculated according to Becker (1984).

Third generation $C$. rosaceana larvae were not raised to pupation, which precluded calculations of parent-offspring regressions of PW and DT using second generation parents or calculations of thirdgeneration full-sib heritabilities for these traits. There was a significant phenotypic correlation in LW in the parents crossed to produce the third generation, despite the fact that they had not been mated assortatively with respect to this trait. This is interesting as significant negative genetic correlations were found 
between DIAP and LW for caterpillars fed on host plants or artificial diet (Carrière, 1991). Consequently, the parent-offspring estimates of heritability in LW obtained using second generation parents were corrected for assortative mating (Falconer, 1981).

\section{Results}

\section{Influence of parental effects on phenotypic means and variances}

The mean diapausing propensities of the first-, secondand third-generation families were $0.70 \pm 0.24$ ( \pm S.D.), $0.17 \pm 0.14$ and $0.17 \pm 0.13$, respectively. Due to 'sampling error' that occurred when the parents were selected to produce a subsequent generation (see Discussion), the mean DIAP within the families of the parents that generated the second generation was $0.52 \pm 0.25$ ( \pm S.D.). The distribution of DIAP within these latter parental families was significantly different from the distribution of DIAP within the families of the second generation (Mann-Whitney, $U=421$, $\chi^{2}=48.45, P<0.001$; Fig. 1). However, the distribution of DIAP within the families of the parents that generated the third generation did not differ from the distribution of DIAP within third-generation families $\left(U=1500, \chi^{2}=1.88, P=0.17\right.$ : mean diapausing pro-

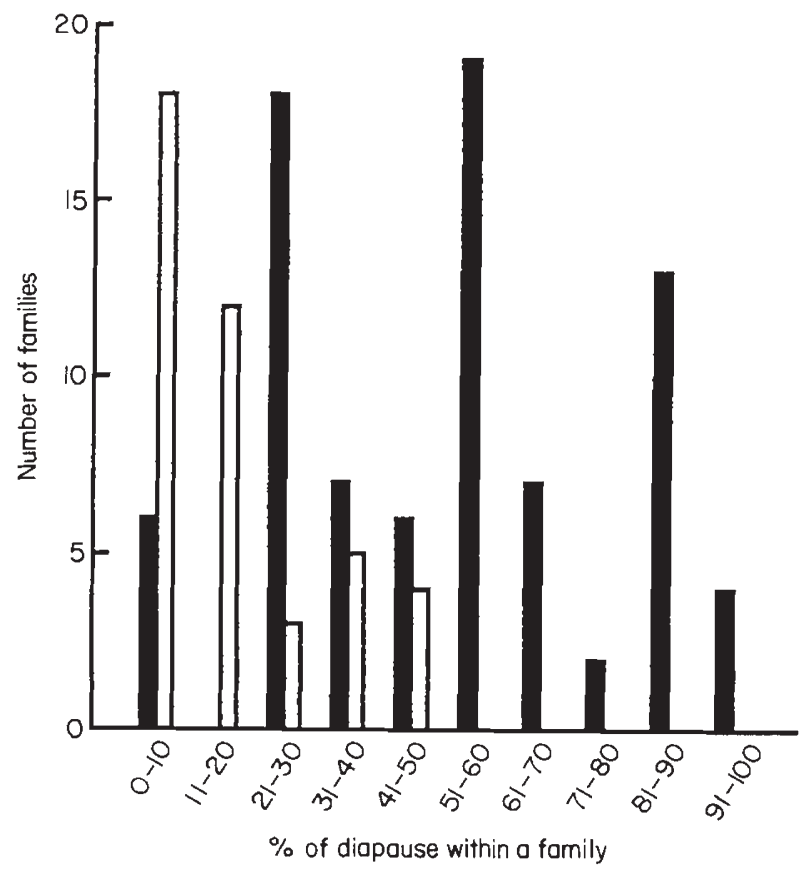

Fig. 1 Distribution of diapausing propensity within the parental families of the first laboratory generation and within the 42 families they produced (the second generation). Solid bars represent the parents; open bars the offspring. pensity in parents, $0.22 \pm 0.18 ; 0.17 \pm 0.13$ in offspring). The observed change in DIAP between the first and second generations and the contrasting lack of change between the second and third generations, suggests that parental effects influenced DIAP in the first generation.

The mean DIAP of larvae produced by spring and summer parents was $0.70 \pm 0.24$ ( \pm S.D.) and $0.38 \pm 0.23$, respectively. The distribution of DIAP within the families of the two groups was significantly different $\left(U=956.5, \chi^{2}=21.87, P<0.001\right.$; Fig. 2). This further indicates the impact of parental effects on diapause incidence in $C$. rosaceana.

Mean live larval weight after 16 days, an index of larval growth rate, was not significantly different between the first- and second-generation larvae (Table 1). Both sexes, however, attained higher PW and males also pupated faster in the first than in the second generation. The phenotypic variance was larger in the first than in the second generation for all traits except DT in males (Table 1). These results suggest that parental effects transmitted by field insects modified the phenotypic mean and variance in the offspring.

Contrary to the first two generations, LW increased significantly between the second and third generations in both sexes. The phenotypic variance also increased in females (Table 1). The cause of these changes is unclear.

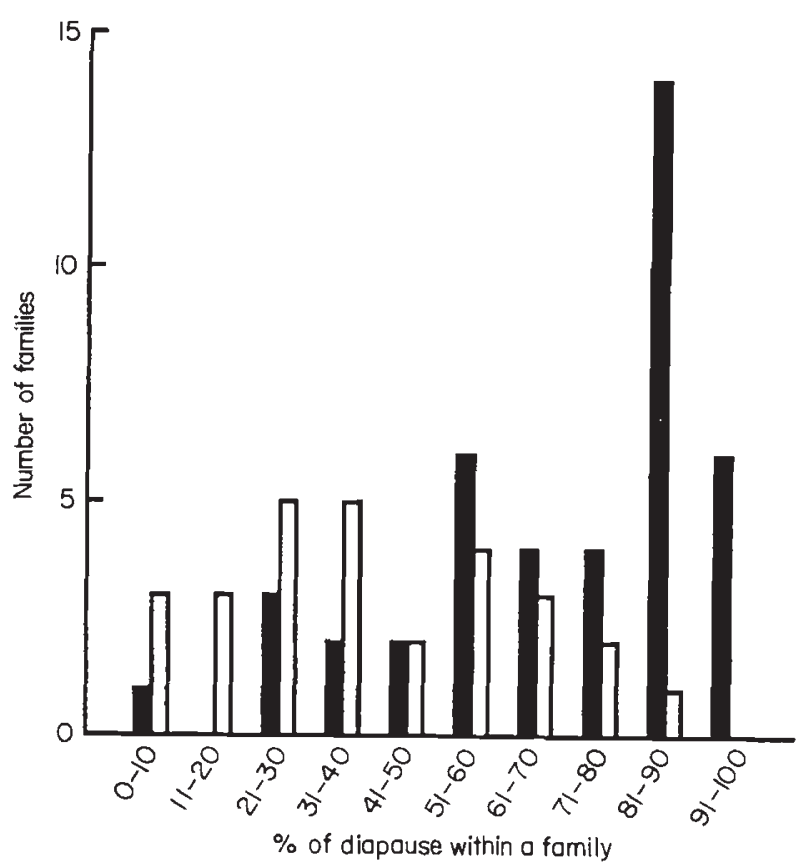

Fig. 2 Distribution of diapausing propensity within the families originating from 'spring' and 'summer' field-raised obliquebanded leafrollers. Solid bars represent offspring produced by 'spring' parents; open bars, 'summer' parents. 
Table 1 Live larval weight after 16 days $(\mathrm{LW}$; in $\mathrm{mg}$, $\ln$ transformed), pupal weight (PW; in $\mathrm{mg}$, ln transformed) and development time (DT; in days) for three generations of leafrollers raised in the laboratory. Means $(\overline{\mathrm{X}})$, standard deviations (s.d.) and sample size $(N)$ are listed by sex

\begin{tabular}{|c|c|c|c|c|c|c|c|}
\hline \multirow[b]{2}{*}{ Sex } & \multirow[b]{2}{*}{ Trait } & \multirow[b]{2}{*}{ Parameter } & \multicolumn{5}{|c|}{ Generations } \\
\hline & & & 1 & & 2 & & 3 \\
\hline \multirow[t]{9}{*}{$\mathrm{M}$} & LW & $\bar{X}$ & 4.27 & ns $\dagger$ & 4.32 & $*$ & 4.56 \\
\hline & & s.d. & 0.82 & $* \ddagger$ & 0.62 & ns & 0.65 \\
\hline & & $N$ & 197 & & 507 & & 506 \\
\hline & PW & $\overline{\mathrm{X}}$ & 4.31 & * & 4.27 & & - \\
\hline & & s.d. & 0.14 & * & 0.12 & & - \\
\hline & & $N$ & 198 & & 510 & & - \\
\hline & DT & $\overline{\mathrm{X}}$ & 21.8 & * & 22.3 & & - \\
\hline & & s.d. & 2.6 & ns & 2.4 & & - \\
\hline & & $N$ & 197 & & 510 & & - \\
\hline \multirow[t]{9}{*}{ F } & LW & $\bar{X}$ & 4.46 & ns & 4.50 & * & 4.71 \\
\hline & & s.d. & 0.84 & * & 0.61 & * & 0.72 \\
\hline & & $N$ & 162 & & 503 & & 488 \\
\hline & PW & $\overline{\mathrm{X}}$ & 4.95 & * & 4.84 & & - \\
\hline & & s.d. & 0.1 .9 & * & 0.14 & & - \\
\hline & & $N$ & 162 & & 500 & & - \\
\hline & DT & $\bar{x}$ & 24.1 & ns & 24.3 & & - \\
\hline & & s.d. & 2.6 & * & 2.2 & & - \\
\hline & & $N$ & 162 & & 503 & & - \\
\hline
\end{tabular}

†Satterthwaite approximate $t$-test of equality of means between generations. ${ }^{*} P<0.05$, ns $=P>0.05$.

$\ddagger$ Folded form of the $F$ statistics to test for homogeneity of variances between generations. ${ }^{*} P<0.05$, ns $=P>0.05$.

\section{Impact of maternal effects on variance components and heritabilities}

With the exception of the among-family variance in development time in males, the among- and withinfamily variance components for $\mathrm{LW}, \mathrm{PW}$ and DT were always greater in the first than in the second generation. A ratio of the among-family components of variance significantly greater than 1 suggests a significant contribution of parental effects through the $V_{\text {ep }}$ term (Table 2). Ratios that were significantly different from 1 for the within-family components of variance suggest that parental effects influenced the withinfamily phenotypic variance through the $V_{\text {ip }}$ term.

The among- and within-family components of variance were always greater in third- than in secondgeneration insects. Only one ratio of the larval weight components was significantly different from 1 (Table 2 ), contrary to the ratios of the first- and second-
Table 2 Between-generation ratios of the among- and within-family components of variance in larval live weight after 16 days $(\mathrm{LW})$, pupal weight $(\mathrm{PW})$ and development time (DT), calculated separately for each sex

\begin{tabular}{ccccc}
\hline \multirow{2}{*}{ Generation } & \multirow{2}{*}{ Sex } & Trait & Among-family & Within-family \\
\cline { 4 - 5 } $1-2$ & M & LW & $2.35^{* *}$ & $1.63^{* *}$ \\
& & PW & 1.38 & $1.52^{* *}$ \\
& & DT & 1.27 & $1.26^{* *}$ \\
& F & LW & $3.14^{* *}$ & $1.66^{* *}$ \\
& & PW & $3.63^{* *}$ & $1.68^{* *}$ \\
$2-3$ & & DT & $2.13^{* *}$ & 1.06 \\
& M & LW & 1.15 & 1.09 \\
& F & LW & 1.24 & $1.36^{*}$ \\
\hline
\end{tabular}

†Ratio significantly different from $1:{ }^{*} P<0.05,{ }^{* *} P<0.01$.

generation components of larval weight that were all significant. This suggests that parental effects generated variation in the variance components.

Although parental effects almost certainly influenced the mean incidence of diapause in the first generation, parent-offspring heritability estimates derived using first-generation parental values were high and similar to the estimates obtained using second-generation parental values (Table 3). Moreover, regressions using either male or female parental values yielded similar heritability estimates as when mid-parental values were used, as expected if parents contribute equally to variation in offspring phenotypes (Falconer, 1981). This suggests the following. (1) Parents raised in the field and in the laboratory transmitted a parental effect of different value. (2) Within a generation, every parent transmitted the same parental effect, in which case the phenotype of a larva (for example its DIAP value in the first generation) would reflect its breeding value (e.g. the DIAP value of its progeny).

Parent-offspring heritability estimates for LW derived using first-generation parental values were not statistically significant, except for the regression of female offspring on female parent (Table 4); however, estimates calculated using second-generation parental values were highly significant. This suggests that heterogeneity in the field conditions resulted in variation in parental effects that reduced the correspondence between the phenotype and breeding value of first-generation parents.

Parent-offspring estimates of heritability derived using first-generation parental values were not significant for PW but parents that had short DT in the first generation produced offspring which developed 
Table 3 Heritability estimates ( \pm S.E.) for within-family propensity of diapause, derived from regressions of offspring family means on single or mid-parent family means

\begin{tabular}{|c|c|c|}
\hline Generations & Regression & $h^{2 \dagger}$ \\
\hline 1,2 & On male parent & $0.39 \pm 0.16$ \\
\hline 1,2 & On female parent & $0.39 \pm 0.16$ \\
\hline 1,2 & On mid-parent & $0.38 \pm 0.10$ \\
\hline 2,3 & On male parent & $0.60 \pm 0.21$ \\
\hline 2,3 & On female parent & $0.51 \pm 0.24$ \\
\hline 2,3 & On mid-parent & $0.38 \pm 0.11$ \\
\hline
\end{tabular}

$\dagger$ All regression coefficients significant at $P<0.02$.

Table 4 Heritability estimates ( \pm S.E.) for live larval weight attained after 16 days, derived from regressions of offspring on one parent, calculated separately for each sex

\begin{tabular}{cccc}
\hline & & \multicolumn{2}{c}{ Parent } \\
\cline { 3 - 4 } Generations & Offspring & Male & Female \\
\hline 1,2 & Male & $0.24 \pm 0.13$ & $0.15 \pm 0.17$ \\
1,2 & Female & $0.20 \pm 0.17$ & $0.28 \pm 0.13^{* \dagger}$ \\
2,3 & Male & $0.54 \pm 0.16^{* * * *}$ & $0.52 \pm 0.15^{* *}$ \\
2,3 & Female & $0.65 \pm 0.13^{* * * *}$ & $0.47 \pm 0.16^{* * *}$ \\
\hline
\end{tabular}

$\dagger$ Significance of the regression coefficients: ${ }^{*} P<0.05$, ** $P=0.0003,{ }^{* * *} P<0.0001$.

rapidly (Table 5). Full-sib heritability estimates were significant for all traits in each generation (Table 6). The full-sib estimates derived in the second generation were less likely to be biased by field-generated parental effects; they were significant for PW, indicating that heritable variation in that trait was present within the populations. Thus, as in the case of LW, the non-significant parent-offspring regression observed for PW was apparently due to the influence of parental effects.

\section{Discussion}

The prediction of short-term evolutionary change in any trait(s) requires the estimation of the form and strength of selection operating on phenotypes and of the underlying genetic variation (covariation) contributing to the phenotypes. The results of this study suggest that non-Mendelian parental effects produced developmental changes in offspring that modified phenotypic means, variances and among- and withinfamily variance of life-history traits in C. rosaceana. Therefore, a quantitative-genetic model describing the
Table 5 Heritability estimates ( \pm S.E.) for pupal weight (PW) and developmental time (DT), derived from regressions of offspring on one parent, calculated separately for each sex. Parents originated from the first laboratory generation

\begin{tabular}{|c|c|c|c|}
\hline \multirow[b]{2}{*}{ Character } & \multirow[b]{2}{*}{ Offspring } & \multicolumn{2}{|c|}{ Parent } \\
\hline & & Male & Female \\
\hline \multirow[t]{2}{*}{ PW } & Male & $0.27 \pm 0.15$ & $0.24 \pm 0.16$ \\
\hline & Female & $0.22 \pm 0.12$ & $0.22 \pm 0.13$ \\
\hline \multirow[t]{2}{*}{ DT } & Male & $0.55 \pm 0.23^{* \dagger}$ & $0.28 \pm 0.15$ \\
\hline & Female & $0.54 \pm 0.20^{*}$ & $0.44 \pm 0.17^{*}$ \\
\hline
\end{tabular}

$\dagger$ Significance of the regression coefficients: ${ }^{*} P<0.025$.

Table 6 Estimates of heritability from full sibs ( \pm S.E.) for live larval weight after 16 days (LW), pupal weight $(\mathrm{PW})$ and development time (DT) calculated for each sex over three generations

\begin{tabular}{ccccc}
\hline & & \multicolumn{3}{c}{ Character $\dagger$} \\
\cline { 3 - 5 } Sex & Generation & LW & PW & DT \\
\hline \multirow{2}{*}{ M } & 1 & $0.52 \pm 0.15$ & $0.46 \pm 0.15$ & $0.38 \pm 0.15$ \\
& 2 & $0.41 \pm 0.10$ & $0.45 \pm 0.10$ & $0.57 \pm 0.11$ \\
& 3 & $0.42 \pm 0.10$ & - & - \\
F & 1 & $0.60 \pm 0.17$ & $0.68 \pm 0.18$ & $0.77 \pm 0.18$ \\
& 2 & $0.37 \pm 0.09$ & $0.35 \pm 0.09$ & $0.47 \pm 0.11$ \\
& 3 & $0.37 \pm 0.10$ & - & - \\
\hline
\end{tabular}

$\dagger F$-tests of full-sib components of variance all significant at $P<0.0003$.

evolution of life-history traits in this species should consider these effects.

\section{Tempora/ variation and life-history evolution}

The effects transmitted by spring parents apparently increased diapausing propensity within the families. They also influenced the mean and variance of lifehistory traits. Such parental effects may have evolved in C. rosaceana as a result of variation in selection across generations and years.

Geometric mean fitness may be maximized by a partial diapausing strategy in insect genotypes that unpredictably face unfavourable conditions, such as early freezing or high predation late in the season (Seger \& Brockman, 1987). Therefore, parental effects influencing diapausing propensity in $C$. rosaceana may have evolved as a result of across-year variation in selection; however, the optimal life-history in a given 
season may not be totally unpredictable. For example, particularly cold springs may increase development time in the first generation and reduce the probability of completing a second generation before encountering inclement fall conditions. Then, the conditions that prevail at the beginning of a season could be 'captured' by spring parents and serve in programming offspring to diapause accordingly. Spring parents also appeared to transmit parental effects that increased populational phenotypic variance for larval growth rate, development time and pupal weight. Unpredictable across-year variation in selection could also favour this increase in phenotypic variance (Kaplan \& Cooper, 1984; Bull 1987).

As all $C$. rosaceana larvae produced by summer parents must enter diapause, the advantage conferred by a partial diapausing strategy only exists for offspring produced by spring parents. Such variation in selection occurring in alternate generations would favour adjustment of diapause through parental effects (or phenotypic plasticity induced by purely environmental cues). Indeed, larvae of $C$. rosaceana produced by summer parents respond to short fall photoperiods and enter diapause (Gangavalli \& Aliniazee, 1985) whereas diapause is influenced by parental effects in larvae produced by spring parents that develop in longer diapause-averting photoperiods. Across-generation variation in selection could also explain the evolution of parental effects that affected mean development time and pupal weight in C. rosaceana.

\section{Repercussion of parental effects on the expression of alleles}

The parents apparently transmitted parental effects that modified both the among- and within-family components of variance of the traits. This contradicts the belief that parental effects generally contribute to variation among offspring family means without affecting within-family phenotypic variance (Falconer, 1981; but see Rossiter, 1991). Incorporating such an influence of parental effects on among- and withingenotype variance may be especially relevant in the context of adaptive change of quantitative traits in unpredictable environments (Kaplan \& Cooper, 1984; Bull, 1987).

Among-family variances tended to increase more than within-family variances in offspring of spring parents. This resulted in estimates of full-sib heritability that generally appeared greater in the first than in subsequent generations (these estimates were biased for larval and pupal weight). This illustrates that parental effects can influence the variance components independently and suggests that there may be a tradeoff between optimizing concurrently phenotypic means and variances across different environments. In particular, an increase in within-genotype variance expressed in a specific environment without an equivalent change in among-genotype variance would reduce the heritability of a trait and in turn diminish the potential of individuals to respond directly to selection on the mean phenotype expressed in that habitat. The increase in among-genotype variance necessary to reduce that constraint would generally not be favoured in the face of stabilizing (or directional) selection (Bull, 1987). Therefore, non-Mendelian mechanisms of inheritance are perhaps more likely to respond to selection favouring a simultaneous increase in genotypic means and variance than Mendelian mechanisms.

Parental effects are a form of phenotypic plasticity in which parental influences on the environment of their progeny affect offspring phenotype (Mousseau \& Dingle, 1991a, b). Phenotypic plasticity can influence traits independently in the same organism (Bradshaw, 1965; Schlichting, 1986). This independence, especially if obtained through the action of regulatory loci, may explain the drastic changes in phenotypic correlations among traits expressed in different environments and facilitates the evolution of adaptive responses of many traits in variable environments (Groeter \& Dingle, 1987; C. D. Schlichting and M. Pigliucci, unpublished data). In $C$. rosaceana, the life-history traits were not influenced in the same way by parental effects. For development time and within-family diapausing propensity, it appears that every parent developing in a specific environment (laboratory or field) transferred the same parental effect. For larval growth rate and pupal weight, different parental effects were apparently transmitted by the field parents. These latter parental effects masked the offspring breeding values.

\section{Mechanisms generating parental effects}

The fact that the parental influences differentially affected the parent-offspring regression of the traits may indicate that distinct parental factors (e.g. hormones transmitted by the mother to the egg) influenced the expression of each trait. Another non-exclusive hypothesis is that the traits differed in their sensitivity to a variation in the level of a single factor transmitted by the parents (i.e. traits were more or less canalized). Such a response could be due to the action of regulatory loci resulting in threshold phenotypic effects (C. D. Schlichting \& M. Pigliucci, unpublished data).

Assuming that a single factor was transmitted by the parents, two models may describe the influence of parental effects on larval growth rate and pupal weight: the parental effects can be viewed as a random factor or as a fixed effect with many levels that is determined 
by some environmental patterning in the conditions generating the parental effects. At this stage, it seems reasonable to hypothesize that variation in larval diapausing conditions (e.g. initiation of diapause in second or third instar) influenced larval development, resulted in physiological variation in spring parents and generated discontinuity in the factor transmitted by these adults to the egg. Under this hypothesis, parent-offspring heritability estimates of larval and pupal weight derived using caterpillars that had parents with similar diapausing history are expected to be significant.

Larvae that initiate diapause in their second instar in the fall may need more time to attain an equal pupal weight $(=$ adult size $)$ in the next season than larvae that diapause in their third instar. Assuming that the time available to complete the second generation is limited, the appropriate trade-off for offspring of the former adult group is to attain a large pupal size and produce many offspring that will have less time to enter diapause in the fall or to pupate at a smaller size and produce less offspring with a higher probability of diapausing successfully. The high variances observed in offspring produced by spring parents suggest that parental effects adjust pupal size, larval growth rate and perhaps female development time in C. rosaceana. This in turn may synchronize reproduction in the cohorts produced by the two groups of parents and preclude the need for parental effects to influence differently diapausing propensity and development time in these two cohorts.

\section{Methodological considerations}

Because offspring were raised in constant conditions, the phenotypic changes observed between generations were attributed to the influence of parental effects. Without replication, it is not possible to establish with certainty that changes in gene frequency due to random sampling of gametes did not contribute to the phenotypic variation between generations. Two types of indirect evidence support the role of parental effects as the major source of variation in this experiment.

Firstly, it is possible to predict the change in mean phenotype in successive generations using the relationship $R=h^{2} \times S$, where $R$ is the predicted change in phenotype between two generations, $h^{2}$ is the heritability of the trait and $S$ is the selection differential (Falconer, 1981). Larval survival was around 98 per cent in each generation (excluding the diapausing larvae) and selection due to larval mortality is considered to be negligible. As the heritability of a trait cannot exceed 1, the observed change in mean phenotype between two generations should not be greater than the selection differential, unless selection is not the only cause of variation. Selection differentials which differ from zero in Table 7 reflect the amount of 'sampling error' that occurred when parents were selected to generate a subsequent generation. For all traits differing significantly in mean phenotype between the first and second generations, the observed changes exceeded, and often were in opposite direction, to the selection differentials. Such changes in mean phenotype are indicative of the influence of parental effects. The similarity of the mean LW between the first and the second generations suggests constancy of the rearing conditions. The observed change in LW between the second and third generations exceeded the selection differentials. The cause of such an increase in larval growth rate is unclear. There are no reasons to believe that these changes were due to environmental variation (i.e. temperature or diet quality) that occurred throughout the experiment.

Secondly, asssuming that genetic variance is totally additive, it is possible to approximate the magnitude of the expected change in genetic variances and population means that would result from random sampling of alleles among many isolated populations (Falconer, 1981; Chap. 15). Then, the within-population decrease in genotypic variance and the increase in variance among population means are expected to be propor-

Table 7 Selection differential and observed change in the mean phenotype of offspring for generations 1,2 and generations 2,3 . The characters are live larval weight after 16 days (LW), pupal weight (PW) developmental time (DT) and propensity of diapause within a family (DIAP). The parameters are given by sex for LW, PW and DT

\begin{tabular}{|c|c|c|c|}
\hline Sex & Character & $\begin{array}{l}\text { Selection } \\
\text { differential }\end{array}$ & $\begin{array}{l}\text { Observed change in } \\
\text { offspring phenotype }\end{array}$ \\
\hline \multicolumn{4}{|c|}{ Generation 1,2} \\
\hline \multirow[t]{3}{*}{$\mathrm{M}$} & LW & +0.02 & +0.05 \\
\hline & PW & +0.01 & $-0.04 *$ \\
\hline & DT & -0.20 & $+0.50^{*}$ \\
\hline \multirow[t]{4}{*}{$\mathrm{F}$} & LW & +0.06 & +0.04 \\
\hline & PW & -0.02 & $-0.11^{*}$ \\
\hline & DT & -0.17 & +0.20 \\
\hline & DIAP & -0.18 & $-0.53^{*}$ \\
\hline \multicolumn{4}{|c|}{ Generation 2, 3} \\
\hline M & LW & -0.01 & $+0.22^{*}$ \\
\hline \multirow[t]{2}{*}{$\mathrm{F}$} & LW & +0.03 & $+0.21^{*}$ \\
\hline & DIAP & +0.05 & 0 \\
\hline
\end{tabular}

*Characters which showed a significant change in mean phenotype between the two generations (from Table 1 and see Results for tests of DIAP). 
tional to the product of the genotypic variance that was present in the base population times $(1-F)$ and $2 F$, respectively, where $F$ is the inbreeding coefficient of the generation considered. The inbreeding coefficients calculated for the second and third generations were 0.00846 and 0.01739 , respectively (Carrière, 1991). Assuming a heritability of one, the calculated change in genotypic variance would be of approximately 1 per cent between the first and second generations. In addition, the predicted variance in means after one generation would be at least 4.4 times smaller than the change observed for any traits between the first and second generations (Carrière, 1991). Therefore, these calculations indicate that sampling effects could not explain the variation in phenotypic means and variances observed in this experiment.

Summer parents were more likely to originate from families with a low propensity to diapause in the spring. This suggests that part of the reduction in the propensity to diapause between offspring produced by spring and summer parents had a genetic basis. However, the variation in the distribution of the propensity to diapause of these latter groups was similar to the change in distribution observed between the first and second laboratory generations. This latter change was unlikely to be due to a variation in gene frequency (as indicated by constancy of DIAP between the second and third generations), which suggests that the main factor influencing diapause in offspring from spring and summer parents was parental effects.

Parental effects were assumed to contribute to the offspring development independently of the parental phenotypes. We expect the parent-offspring regressions derived using first- and second-generation parental values to yield similar estimates when the parental effects are proportional to the parental phenotype (see Janssen et al., 1988). The pattern of changes in values of parent-offspring regressions observed in this study suggests that parental effects are transmitted independently of the parental phenotype in C. rosaceana.

In general, animals exploiting environments that change over periods that are short relative to their generation time are expected to evolve mechanisms to reduce temporal variance in fitness (Seger \& Brockman, 1987). In this context, parental effects could be seen as a form of plasticity that allows animals to respond appropriately to these changes. Field studies are necessary to assess the commonness of parental effects, the ecological context favouring their evolution and their role in evolutionary biology.

\section{Acknowledgements}

I am very grateful to Sara Paré and Don Thomson for their assistance in this study, as well as to the staff of the Summerland Agriculture Canada research station for their collaboration. I also thank Liz Boulding, Tim Mousseau and Derek Roff for providing helpful comments on previous versions of the manuscript. This study was supported by a Natural Sciences and Engineering Research Council of Canada grant to Bernard Roitberg and a NSERC post-graduate scholarship to Yves Carrière.

\section{References}

BECKER, w. A. 1984. Manual of Quantitative Genetics. Academic Enterprise, Pullman, WA.

BRADSHAW, A. D. 1965. Evolutionary significance of phenotypic plasticity in plants. Adv. Genet., 13, 115-155.

BULl, J. J. 1987. Evolution of phenotypic variance. Evolution, 41, 303-315.

CARRIÈRE, Y. 1991. Ecological Genetics of Host Exploitation in a Generalist Herbivore, the Obliquebanded Leafroller. Ph.D. Thesis, Simon Fraser University.

CARrière, Y. 1992. Host plant exploitation within a population of a generalist herbivore, Choristoneura rosaceana. Entomol. Exp. Appl., 65, 1-10.

CARRIËRE, Y. AND ROITBERG, B. D. 1994. Trade-offs in responses to host plants within a population of a generalist herbivore, Choristoneura rosaceana (Lepidoptera: Tortricidae). Entomol. Exp. Appl., (in press).

CHAPMAN, P. J. AND LIENK, S. E. 1971. Tortricid Fauna of Apple in New York. Special publication. New York State Agricultural Experimental Station Geneva, NY.

FALCONER, D. S. 1981. Introduction to Quantitative Genetics 2nd edn. Longmans, New York.

GANGAVALLI, R. R. AND ALINIAZEE, M. T. 1985. Diapause induction in the obliquebanded leafroller Choristoneura rosaceana (Lepidoptera: Tortricidae): role of photoperiod and temperature. J. Insect Physiol., 31, 831-835.

GIESEL, J. T. 1986. Genetic correlation structure of life history variables in outbred, wild Drosophila melanogaster: effects of photoperiod regimen. Am. Nat., 128, 593-603.

GIESEL, J. T. 1988. Effects of parental photoperiod on development time and density sensitivity of progeny of Drosophila melanogaster. Evolution, 42, 1348-1350.

GOMULKIEWICZ, R. AND KIRKPATRICK, M. 1992. Quantitative genetics and the evolution of reaction norms. Evolution, 46, 390-411.

GROETERS, F. R. AND DINGLE, H. 1987. Genetic and maternal influences on life history plasticity in response to photoperiod by milkweed bugs (Oncopeltus fasciatus). Am. Nat., 129, 332-346.

GUPTA, A. R. AND LEWontin, R. C. 1982. A study of reaction norms in natural populations of Drosophila pseudoobscura. Evolution, 36, 934-948.

HARDIE, J. AND LEE, A. D. 1985. Endocrine control of polymorphism and polyphenism. In: G. A. Kerkuk and L. I. Gilbert (eds) Comprehensive Insect Physiology, Biochemistry and Pharmacology, vol. 8, pp. 441-490. Pergamon Press, Toronto. 
HOLLOWAY, G. J., POVEY, S. R. AND SIBLY, R. M. 1990. The effect of new environment on adapted genetic architecture. Heredity, 64, 323--330.

JANSSEN, G. M., DE JONG, G., JOOSSE, E. N. G. AND SCHARLOO, W. 1988. A negative maternal effect in springtails. Evolution, 42, 828-834.

KAPLAN, R. H. AND COOPER, w. S. 1984. The evolution of developmental plasticity in reproductive characteristics: an application of the 'adaptive coin-flipping' principle. Am. Nat., 123, 393-410.

KIRKPATRICK, M. AND LANDE, R. 1989. The evolution of maternal characters. Evolution, 43, 485-503.

LANDE, R. 1979. Quantitative genetic analysis of multivariate evolution, applied to brain: body size allometry. Evolution, 33, 402-416.

LEVINS, R. 1968. Evolution in Changing Environments, Princeton University Press, Princeton, NJ.

MOUSSEAU, T. A. 1991. Geographic variation in maternalage effects on diapause in a cricket. Evolution, 45, 1053-1059.

MOUSSEAU, T. A. AND DINGLE, H. 1991a. Maternal effects in insect life histories. Ann. Rev. Entomol., 36, 511-535.

MOUSSEAU, T. A. AND DINGLE, H. $1991 \mathrm{~b}$. Maternal effects in insects: examples, constraints and geographic variation. In: Dudley, E. C. (ed.) The Unity of Evolutionary Biology, The Unity of Evolutionary Biology: Proceedings of the ICSEB IV Meeting, pp. 745-761. Dioscorides Press, Portland, OR.

NEWMAN, R. A. 1988. Adaptive plasticity in development of Scaphiopus couchii tadpoles in desert ponds. Evolution, 42, 774-783.

RAUSHER, M. D. 1985. Competition, frequency-independent selection and diapause in Battus philenor butterflies. Flor. Entomol., 69, 62-78.

ROFF, D. A. 1986. The genetic basis of wing dimorphism in the sand cricket, Gryllus firmus and its relevance to the evolution of wing dimorphism in insects. Heredity, 57, 221-231.

ROFF, D. A. 1990. Selection for changes in the incidence of wing dimorphism in Gryllus firmus, Heredity, 65, 163-168.

ROFF, D. A. 1994. Evolution of dimorphic traits: effect of directional selection on heritability. Heredity, (in press).

Rossiter, M. C. 1991. Maternal effects generate variation in life history: consequences of egg weight plasticity in the gypsy moth. Funct. Ecol, 5, 386-393.

SAS INSTITUTE INC. 1988. SAS Users' Guide: Statistics Version 6.03. SAS Institute Inc, Cary, NC.

SCHEINER, S. M., CAPLAN, R. L. AND LYMAN, R. F. 1989. A search for trade-offs among life history traits in Drosophila melanogaster. Evol. Ecol., 3, 51-63.

SCHLICHTING, C. D. 1986. The evolution of phenotypic plasticity in plants. Ann. Rev. Ecol. Syst., 17, 667-693.

SCHMALhausEn, I. I. 1949. Factors of Evolution: The Theory of Stabilizing Selection. Blakiston, Philadelphia.

SEGER, J. AND BROCKMANN, H. J. 1987. What is bet-hedging? In: Harvey, P. H. and Partridge, L. (eds) Oxford Surveys in Evolutionary Biology, vol. 4, pp. 182-211. Oxford University Press, Oxford

SHOREY, H. H. AND HALE, R. C. 1965. Mass rearing of the larvae of nine noctuid species on a simple artificial medium. $J$. Econ. Entomol., 58, 522-524.

SOKAL, R. R. AND ROHLF, F. J. 1981. Biometry, 2nd edn. Freeman and Co, New York.

STEARNS, S. C. 1989. The evolutionary significance of reaction norms. Bioscience, 39, 436-446.

STEARnS, S. C. 1992. The Evolution of Life Histories. Oxford University Press, Oxford.

TAUBER, C. A. AND TAUBER, M. J. 1986. Genetic variation in allor-none life-history traits of the lacewing Chrysoperla carnea. Can. J. Zool., 64, 1542-1544.

VAN NOORDWIJK, A. J. AND GEBHARDT, M. 1987. Reflections on the genetics of quantitative traits with continuous environmental variation. In: V. Loeschcke (ed.) Genetic Constraints on Adaptive Evolution, pp. 73-90. Springer Verlag, Berlin.

VAN TIENDEREN, P. H. 1991. Evolution of generalists and specialists in spatially heterogeneous environments. Evolution, 45, 1317-1331.

vIA, S. 1987. Genetic constraints on the evolution of phenotypic plasticity. In: V. Loeschcke, (ed.) Genetic Constraints on Adaptive Evolution, pp. 47-71. Springer Verlag, Berlin.

vIA, S. 1993. Adaptive phenotypic plasticity: target or byproduct of selection in a variable environment? Am. Nat, 142, 352-365.

VIA, S. AND LANDE, R. 1985. Genotype-environment interaction and the evolution of phenotypic plasticity. Evolution, 39, 505-523. 